\title{
La prensa digital y las redes sociales en la actividad 2.0 de los menores gallegos
}

\section{Digital press and social networks in the 2.0 activity of the Galician children}

\author{
Beatriz Feijoo Fernández \\ Investigadora predoctoral del Departamento de \\ Psicología Evolutiva y Comunicación \\ (Universidade de Vigo)
}

Fecha de recepción: 30 de septiembre de 2014

Fecha de revisión: 15 de enero de 2015

Para citar este artículo: Feijoo-Fernández, B. (2015): La prensa digital y las redes sociales en la actividad 2.0 de los menores gallegos, Icono 14, volumen (13), pp. 30-51. doi: 10.7195/ri14.v13i1.739 


\section{Resumen}

Este artículo tiene como objetivo analizar la actividad digital de los niños gallegos, concretamente su nivel de visitas a la prensa digital y a las redes sociales. Ambos espacios web no fueron diseñados en un principio para un público infantil, lo que no es impedimento para que sean recurrentes en las rutinas de navegación de los más pequeños, aunque para ello tengan que incumplir las condiciones legales de las webs, como ocurre en las redes sociales, que restringen el acceso a menores de 14 años. A través de un análisis cuantitativo se busca comparar el uso que este tipo de usuarios hacen de la prensa digital y de las redes sociales, concretando cómo es su 'relación' en el momento actual y cuáles son sus webs favoritas.

\section{Palabras clave}

TIC - Infancia - Comunicación - Nativos digitales

\section{Abstract}

Children have become an important potential public to the networked entertainment market. They are digital native and their natural relationship with new technologies make that their presence in the net is higher than people of other ages. In relation with this, the mass media, the digital press specifically, is trying to attract the children attention with educational strategies to turn them in press's readers and in this way increasing its users. However, there are other online services which are not designed to infancy, but they have the minor's favor: an example is the social networks. In this way, this article deepens in the concept of infancy as digital user to analyze the results of a poll which was replied by children of 11/12 years old to check and compare the penetration level of the digital press and social networks in in children's media habits thanks to a sample of 2200 individuals.

\section{Key Words}

Digital press - Social networks - Children - Internet habits - Digital natives 


\section{Introducción}

Los niños y niñas siempre han sido un público a tener en cuenta para el mercado de entretenimiento en internet. Su condición de nativos digitales y su relación natural con las nuevas tecnologías provocan que su presencia en la red sea mayor en proporción que la de otros grupos de edad. En esta línea, medios como la prensa digital, conocedor del tiempo de conexión de la infancia, tratan de atraer su atención con estrategias y proyectos educativos para fidelizarlos como lectores y así incrementar sus números de usuarios, algo imprescindible para mejorar su impacto y ser atractivo para los anunciantes.

Sin embargo, hay otro tipo de servicios digitales, que sin ser concebidos específicamente para la infancia, cuentan con su más absoluto seguimiento, como son las redes sociales. Es más, muchos de los menores son usuarios de las mismas sin tener la edad mínima para acceder, 14 años en España. Así, un estudio realizado por Consumer Reports (http://www.consumerreports.org/) estimaba en 2010 que la cantidad de usuarios de 13 años o menos en Facebook era más de 7,5 millones, de los cuales cinco millones tenían 10 años o menos.

Ciertamente, este vínculo entre jóvenes y nuevas tecnologías cada vez se da en edades más tempranas. Son los nuevos miembros de una categoría social que Marc Prensky ya definía en 2001 como "nativos digitales". La forma de interactuar con estos soportes, su omnipresencia en sus rutinas diarias provocan grandes diferencias con el otro grupo social establecido por Prensky en oposición a los nativos digitales, los "inmigrantes tecnológicos", "personas que no han nacido inmersas en este entorno de nuevas tecnologías, pero que se ven obligadas a utilizarlas", según Bringué y Sádaba (2009:16). Si los primeros mantienen una relación natural con las TIC, "son su lengua materna", para los segundos "son una lengua extranjera". Para Bringué y Sádaba (2009:16) esta brecha digital provocada principalmente por la variable edad repercute de forma acusada en el sector educativo y familiar, pues padres y docentes se sienten perdidos en el mundo de los nuevos medios en el que sus hijos les sacan mucha ventaja. De ahí que en los últimos años se incrementasen los estudios científicos sobre infancia y su uso de las TIC con el fin de dar respuesta a un sinfín de interrogantes.

DOI: ri14.v13i1.739 | ISSN: 1697-8293 | Año 2015 Volumen 13 Nº 1 | ICONO14 
En este sentido, este artículo se centra en el usuario infantil en el contexto digital para comparar el nivel de penetración de la prensa online y de las redes sociales en las rutinas de navegación de este tipo de usuarios. Se trata de comprobar si las acciones de los diarios para acercarse a la infancia son efectivas y ganan notoriedad en su lista de sitios web más visitados, entre los que se encuentran las redes sociales.

\section{Marco teórico y contexto}

\subsection{La prensa digital}

\subsubsection{Datos y perfil de su audiencia}

El Libro Branco de la Prensa Diaria 2013, editado por la Asociación de Editores de Diarios Españoles (EADE), confirma que los medios online han alcanzado en cifras de difusión a los tradicionales: 12 millones de lectores frente a los 13 millones de los impresos. Frente al descenso de audiencia de la prensa de papel en este período- según 0JD ha perdido un 12,6\% de difusión en los primeros siete meses de 2013- la prensa online duplicó su número de usuarios desde 2009, al incrementar su audiencia en un 114\%, tal como recoge la segunda ola de 2013 del Estudio General de Medios (EGM).

Como apunta el Libro Blanco de la Prensa en su edición de 2013, la prensa digital representa casi el $10 \%$ del total de inversión publicitaria en prensa. En el año 2011 los diarios offline retrocedieron un 12\% la inversión publicitaria- pasando de 1.057,4 a 930,9 millones de euros-, mientras que la prensa online aumentó la inversión publicitaria un 10,9\% - creciendo de 106,7 en 2010 a 118,3 millones de euros en 2011-. Los diarios digitales ayudaron a suavizar la disminución de los ingresos publicitarios en el conjunto total del medio, que retrocedieron un $10 \%$.

A esta tendencia opuesta entre diarios digitales e impresos también se suman diferencias en el perfil de lector. Aquel que opta por la red suele ser un tipo de lector más atractivo para los anunciantes por tres motivos, uno generacional, dos educativo y tres por su poder adquisitivo. Mientras que la prensa de papel se enfrenta al envejecimiento de sus compradores, la digital presenta un perfil más joven, con mayor nivel de estudios y de ingresos económicos.

ICONO14 | Año 2015 Volumen $13 N^{\circ} 1$ | ISSN: 1697-8293 | DOI: ri14.v13i1.739 
La segunda ola de 2013 del informe sobre la Audiencia de Internet del EGM, que proporciona información referida exclusivamente a los sitios web que audita y controla, situó a quince diarios digitales como los espacios más visitados en la red: Marca, El País, As, 20minutos, Sport, ABC y La Vanguardia a la cabeza, seguidos de otros como El Mundo Deportivo, El Periódico, El Economista, Expansión, La Razón, La Voz de Galicia y Cinco Días. La auditación de septiembre 2013 de 0JD también registra como medios más visitados a Marca.com, El Mundo y 20minutos.es. No obstante, el EGM refleja un descenso de los usuarios de lectura de información de actualidad en internet con respecto a 2012.

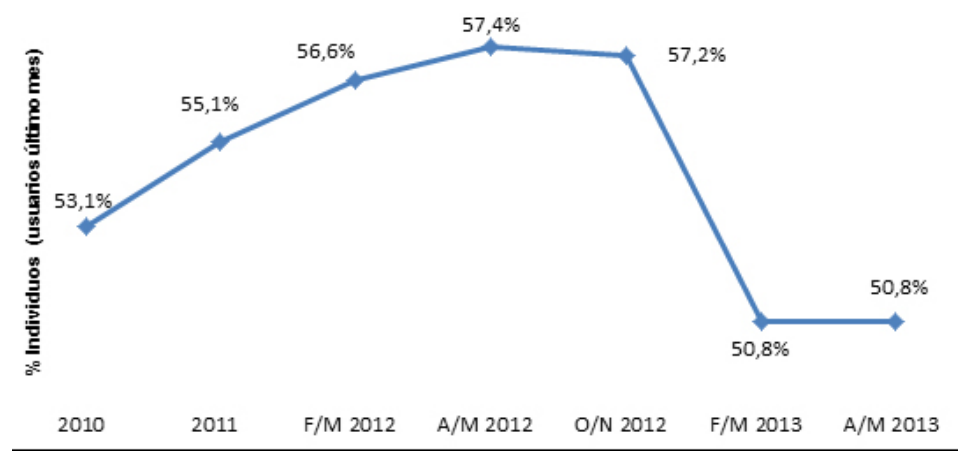

Figura 1: Usuarios de 'lectura de información de actualidad' en internet. Fuente: EGM, 2013

\subsubsection{Audiencia infantil y prensa digital}

Dada su condición de nativos digitales (Prensky, 2001) y de sus niveles de uso de la red, niños y niñas pueden ser un público atractivo para la prensa digital, interesada en buscar nuevos nichos de mercado y por crecer en número de usuarios. En esta línea, en Europa es común la existencia de cabeceras online dirigidas específicamente a los menores, como el semanario alemán Kruschel (http://www.kruschel.de/) con 2.100 suscripciones, o en Reino Unido First News (http://www.firstnews.co.uk/), concebido para niños y niñas de nueve a 12 años y que en 2012 ha crecido un 18\% su difusión, lo que supone una media de dos millones de lectores cada semana, según datos publicados por el medio digital Media-tics. Que estas publicaciones estén orientadas a la infancia no significa que eludan temas complejos, así desde sus primeros números, el semanario inglés ha tratado aspectos como las guerras de Irak o Afganistán. 
Esta potenciación de la relación entre prensa e infancia también se produce en España. Hay ejemplos de cabeceras nacionales que tienen o tuvieron en marcha proyectos educativos para integrar la prensa en la escuela, pero orientados más bien como parte de una estrategia de responsabilidad corporativa que como una campaña de captación de nuevos públicos.

En este caso se toma como referente la iniciativa impulsada por el diario gallego La Voz de Galicia, llamada Prensa Escuela, una apuesta por introducir el periódico en el aula y convertirlo en un elemento didáctico y de aprendizaje para los alumnos. Este proyecto educativo se puso en marcha en 1982 en forma de suplemento semanal que se repartía con todas las ediciones impresas del diario. Desde 2005 cuenta con una plataforma online para introducir en el uso constructivo de nuevas tecnologías a los 42.500 alumnos, 1.200 profesores y 550 centros de toda Galicia que participan en la iniciativa.

Así Prensa Escuela dispone de una página web (http://www.prensaescuela.es/) a partir de la cual los escolares pueden crear su propia bitácora y ejercer la profesión de periodistas, siempre supervisados por un profesor responsable. En la red también tienen activados otros servicios como cursos para docentes, concursos para incentivar la participación de los alumnos, material didáctico para ayudar al profesor a utilizar la noticia en el aula, o secciones como Pregunta a un Científico - Pregunta a un Periodista, en las que los niños plantean curiosas cuestiones respondidas por los periodistas y expertos que trabajan para el diario.

Este tipo de acciones pueden ayudar a promocionar el medio en otros sectores de la sociedad, en este caso en la escuela, un campo atractivo en el que experimentar para garantizar lectores futuros. La prensa y en mayor medida la impresa, ve amenazado su crecimiento por un cierto desinterés de los más jóvenes hacia la lectura, por lo que estos programas pueden ser una buena vía para paliar esa apatía por el medio.

Son numerosos los investigadores (Romano, 2003; Ricoy, 2006; Buil, Hernández y Sesé, 2013) los que justifican la integración de los medios de comunicación en las escuelas pues desempeñan "una función educativa relevante" (Ricoy, 2009:147). 
Álvarez (citado en Buil et al., 2013) destaca tres razones fundamentales por las que los mass media deben tenerse en cuenta en los currículos académicos:

En primer lugar, las características de las informaciones que en ellos se recogen, como serían la actualidad, la diversidad, la pluralidad o la presentación de problemas reales. En segundo lugar, su relevancia en la formación crítica entre los estudiantes. El empleo de los medios de comunicación como recurso educativo fomenta el desarrollo de competencias tan relevantes como la búsqueda, interpretación y uso de la información, o el desarrollo del sentido crítico. En tercer lugar [...] la introducción de los medios de comunicación en la educación se alinea con la ruptura de un modelo de enseñanza puramente transmisivo, centrado en los conocimientos y el profesor. (Buil et al., 2013:447-448).

Concretamente la prensa, tanto en su formato impreso como digital, se presenta como el medio idóneo para desarrollar las competencias mencionadas. Es versátil y de fácil acceso en las aulas, por tanto cercano a su rutina, lo que favorece la conexión de los contenidos tratados en el aula con la realidad y al mismo tiempo se traduce en una mayor motivación del estudiante (Aguaded y Pérez, 2000, citado en Buil et al., 2013). Actualizar los contenidos de los libros de texto o de otras fuentes académicas, la transmisión de valores, la potenciación de una visión crítica (Ricoy, 2009), el enriquecimiento verbal y cultural o la toma de consciencia de lo que está sucediendo alrededor son otros beneficios que la prensa puede aportar a los escolares y enriquecer así su potencial formativo.

\subsection{Las redes sociales}

\subsubsection{Volumen y perfil de usuarios}

El V Estudio anual sobre Redes Sociales, que elabora la organización empresarial de publicidad y marketing online IAB Spain Research en colaboración con Elogia Media, ofrece una descripción general del panorama digital en 2013 y destaca un mercado que ya ha alcanzado su estado de madurez. Según datos de dicho informe, ocho de cada 10 internautas emplean redes sociales, un nivel de alcance que se mantiene estable si se compara con datos del año anterior.

DOI: ri14.v13i1.739 | ISSN: 1697-8293 | Año 2015 Volumen 13 Nº 1 | ICONO14 


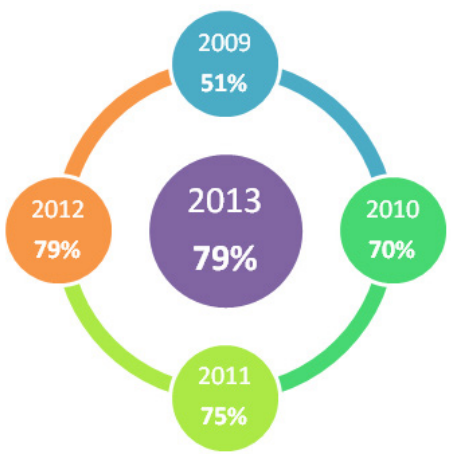

Figura 2: Evolución de la penetración de las redes sociales en España. Fuente: V Estudio anual sobre Redes Sociales. Elaboración propia, 2014

Según datos que hace público la empresa Comscore a través de su informe 2013 Spain digital future in focus, Facebook tiene 17 millones de usuarios únicos (conectados desde PC) en España a diciembre de 2012, 200 mil menos que en 2011. Igualmente Tuenti pierde un millón de usuarios activos, bajando hasta los 9,6 millones. Por su parte, Linkedin alcanza los 2,7 millones de usuarios y Twitter 5,6 millones, creciendo ambas en más de un millón con respecto al año anterior.

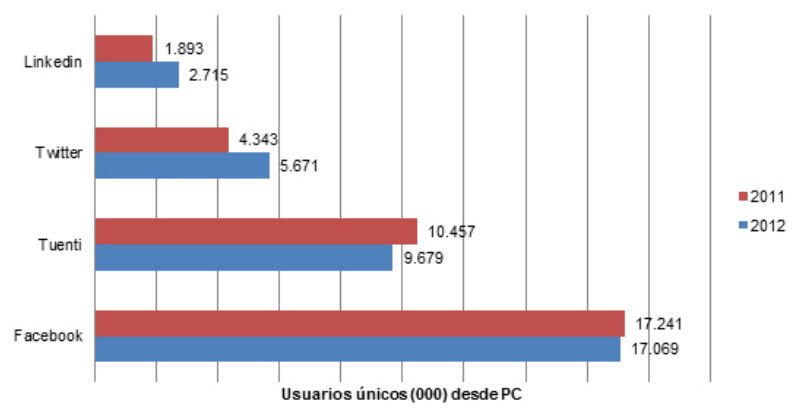

Figura 3: $N^{0}$ usuarios desde PC de redes sociales en España. Fuente: Comscore, 2013

Para los analistas, es destacable el aumento significativo del acceso a redes sociales desde el teléfono móvil. Siguiendo datos facilitados por Comscore, el número de usuarios únicos conectados desde el móvil a Twitter y a Linkedin se duplicaron en el plazo de un año en España. En Facebook se incrementaron en algo más de un 50\%, pasando de 8,6 millones a 13,8 millones de usuarios móviles; Tuenti aumentó en un millón este tipo de conexiones. 


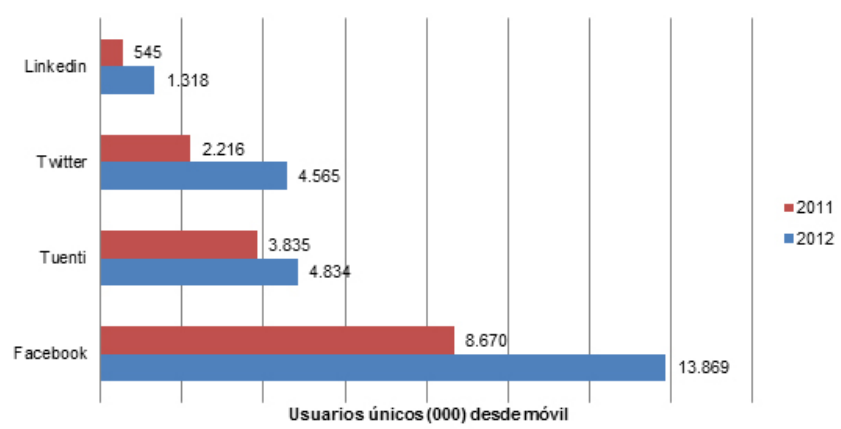

Figura 4: No usuarios móviles de redes sociales en España. Fuente: Comscore, 2013

La frecuencia de acceso a las redes sociales suele ser diaria, un nivel de uso que ya era mayoritario en 2012. Facebook es la que se visita un mayor número de veces además de ser a la que más horas dedican los internautas a la semana.

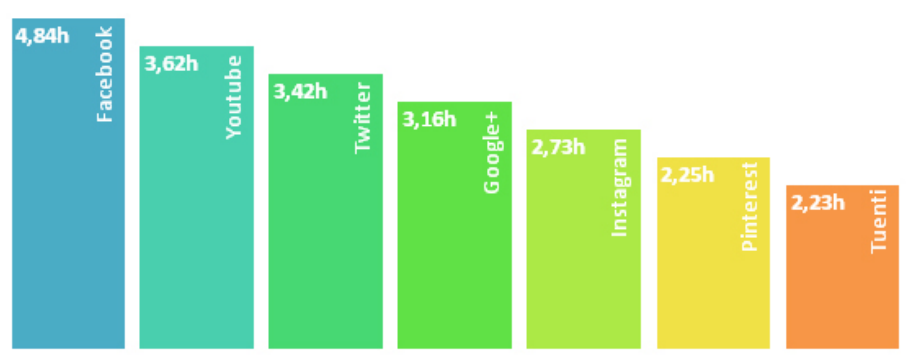

Figura 5: Horas dedicadas a la semana a las rrss. Fuente: V Estudio anual sobre Redes Sociales. Elaboración propia, 2014

El usuario tipo de las redes sociales es un individuo de 36 años, trabajador por cuenta ajena. Con el paso de los años se han ido reduciendo las diferencias de uso en función del sexo por lo que hombres y mujeres tienden a tener una presencia similar en dichas aplicaciones. Los internautas que no emplean las redes sociales declaran no hacerlo por una cuestión de desinterés sobre el contenido de las redes sociales y por una falta de protección de su privacidad. Acostumbra ser un público ligeramente más adulto que el usuario de redes sociales. 
En relación al uso dado a las redes sociales, se acentúa el perfil observador: un $66 \%$ de los individuos afirma que su principal actividad es revisar la actividad -fotos, vídeos, actualización de estados y noticias- de sus contactos. También prima la comunicación mediante el intercambio de mensajes (59\%) y el uso del chat (46\%). Consumir contenido, sobre todo audiovisual, es una actividad recurrente para la mitad de los usuarios, mientras que otras rutinas más interactivas, como publicar contenidos, comentar actualidad o jugar, son desempeñadas en menor medida $(30 \%)$.

Como prospectiva de futuro, el uso de las redes sociales tenderá cada vez más hacia la especialización, empleando una u otra red para un fin concreto. De este modo, para consumo de música y vídeo, despuntarán Spotify y Youtube; para comunicarse o conocer gente, Badoo, MySpace o Tuenti; para un uso profesional, Linkedin, Twitter o Google+ o para participar en concursos, Facebook o Twitter.

\subsubsection{El usuario infantil y las redes sociales}

Una de las principales fuentes de información en España sobre el estudio científico de las redes sociales en la vida de los menores es El Foro Generaciones Interactivas (http://www.generacionesinteractivas.org), una organización sin ánimo de lucro integrada por profesionales de los ámbitos académico, docente y empresarial cuya misión es "promover un uso de la tecnología que haga mejores a las personas", (Bringué y Sádaba, 2011:10). Fundada en diciembre de 2008, se trata de un organismo promovido por Telefónica, la Universidad de Navarra y la Organización Universitaria Interamericana (OUI).

Al amparo de este foro vio la luz en 2011 el estudio Menores y redes sociales, que a partir de trece mil encuestas realizadas a menores de entre 10 y 18 años concluía que un $71 \%$ de la muestra (9.230 individuos) utiliza redes sociales y que a partir de los 14 años dicho uso supera el $80 \%$ hasta alcanzar su cota máxima a los 17 años con un 85\% de penetración, (Bringué y Sádaba, 2011:24)

La red social más popular entre este público es Tuenti, seguida a gran distancia por Facebook, en una proporción de tres a uno a favor de la primera, (García

ICONO14 | Año 2015 Volumen $13 N^{\circ} 1$ | ISSN: 1697-8293 | DOI: ri14.v13i1.739 
Fernández, 2010:25-26). A medida que se hacen mayores, aumenta su interés por Facebook a la vez que decrece el de Tuenti. García Fernández (2010:26) pone de relevancia que, a pesar de que muchas de las redes sociales mencionadas prohíben en sus condiciones de uso el acceso a menores de 14 años- como Tuenti- son muchos los niños y niñas de edad inferior dados de alta en alguna de estas redes sociales.

Tener perfil y visitar redes sociales influye en el tiempo dedicado a internet; los menores que están dados de alta en estas aplicaciones están conectados durante más tiempo, (García Fernández, 2010:36). Entre otras cuestiones, la relación entre contenidos visitados y uso de redes sociales es opuesta en lo referido a contenidos de tipo educativo y cultural, "los que no usan redes sociales suelen visitarlos con mayor frecuencia, especialmente en el caso de las chicas", (García Fernández, 2010:37). Para el investigador es preocupante que, en lo referido al control parental, apenas dos de cada 10 que usan redes sociales tengan restricciones en el momento de colgar fotos y vídeos.

En 2008 Bringué y Sádaba comenzaron a emplear el término “Generaciones Interactivas" para referirse a estos niños y niñas "cuya existencia se explica desde la convivencia habitual con los múltiples dispositivos propios de las Tecnologías de la Información y de la Comunicación (TIC)", (Bringué y Sádaba, 2009:315). A grandes rasgos, la definen como una generación que vive en hogares plenamente equipados con la mayoría de soportes disponibles en el mercado; precoz, con acceso a las pantallas y uso intensivo de las mismas antes de los 10 años; móvil, ya que a edades tempranas cuentan con smartphones que les permiten estar online independientemente del lugar; consciente de forma desigual de los riesgos que corren en este mundo virtual; autónoma y autodidacta, que accede libre e independientemente a las pantallas; multitarea, con la necesidad constante de hacer 0 atender varias cosas a la vez y con una visión multifuncional de la tecnología. En función del sexo, los niños consideran las pantallas un medio a través del cual desarrollar actividades de acción (videojuegos, juegos online...); por el contrario, las niñas prefieren el uso relacional y optan por chats e interaccionar con los demás.

DOI: ri14.v13i1.739 | ISSN: 1697-8293 | Año 2015 Volumen 13 Nº 1 | ICONO14 
Las redes sociales son atractivas para este público (García Fernández, 2010:43) porque le permiten interaccionar, socializar con sus iguales, crear espacios personales y privados a los que no tienen acceso sus adultos y acceder a un volumen de información ilimitado.

Los menores se han volcado con entusiasmo en las redes sociales por las posibilidades de comunicación que les dan estas plataformas, (Monsoriu, 2009:4). "Primero, los programas de mensajería, más tarde los blogs y desde hace tres años, las redes sociales" (Monsoriu, 2009:59). En estas etapas se busca la aceptación del grupo de iguales, que a través de la red es más fácil conseguirlo, "por eso se han entusiasmado con las mismas y son los usuarios mayoritarios" (Monsoriu, 2009:75). Estos nativos digitales pasan por un momento vital en el que forman su identidad a través de la pertenencia a un grupo social, de ahí que sean tan importantes para ellos dichas plataformas online.

Según la Agencia Española de Protección de Datos, se debe ser mayor de 14 años para disponer de las condiciones de madurez precisas para consentir, por sí mismo, el tratamiento automatizado de sus datos de carácter personal. Al respecto, el Reglamento de Desarrollo de la Ley Orgánica 15/1999, de 13 de diciembre de Protección de Datos de Carácter Personal, establece en su artículo 13 que en el caso de menores de 14 años, para proceder al tratamiento de sus datos, se requerirá consentimiento de padres o tutores.

Desde instituciones como INTECO (Instituto Nacional de Tecnologías de la Comunicación) se insta a las plataformas de networking a que instalen sistemas que garanticen la comprobación de la edad de los usuarios y la autenticidad del consentimiento prestado por los padres o tutores legales si así se requiere (2010:27). Tuenti por ejemplo ya ha puesto en marcha alguna que otra medida para eliminar los perfiles sospechosos de ser menores de 14 años: la red social les envió una solicitud para que aportasen fotocopia del DNI o pasaporte en un plazo de 90 horas, si no se recibía respuesta, directamente se borraba dicho perfil. También están implantando medidas para evitar que determinados contenidos sean mostrados a aquellos usuarios que, en el registro, hubieran indicado su minoría de edad.

ICONO14 | Año 2015 Volumen $13 N^{\circ} 1$ | ISSN: 1697-8293 | DOI: ri14.v13i1.739 


\section{Material y métodos}

Prensa digital y redes sociales son dos servicios presentes en mayor o menor medida en la vida de los niños. Este artículo busca conocer y comparar sus niveles de penetración entre niñas y niños gallegos de 11-12 años que ya realizan un consumo importante y destacado de internet.

Los resultados de este estudio se extraen de una investigación de mayor envergadura llevada a cabo en 2010 para conocer la relación de los niños gallegos con diferentes pantallas (televisión, videojuegos e internet) en la que se empleó como metodología la encuesta supervisada. Se diseñó un sistema de muestreo combinado y bietápico en el que se trabajó con dos unidades de muestro distintas en cada una de las fases: la unidad de muestreo primaria se correspondió con el centro de enseñanza y la unidad de muestreo secundaria fue el individuo de 11 a 12 años, centrándonos en el curso $6^{\circ}$ de primaria, ya que es el año escolar en el que conviven el mayor número de alumnos de dicha edad y ser estos especialmente sensibles desde el punto de vista de generación de una mentalidad de consumo.

La selección de un curso de primaria en concreto motivó la elección de un muestreo aleatorio por conglomerados (D`Ancona, 1996), lo que permitió realizar las agrupaciones de individuos por centro de estudio y curso. Así, en la primera fase se realizó una muestra representativa de colegios, atendiendo a un criterio de proporcionalidad con respecto al universo de estudio, en función del ámbito geográfico (urbano, semiurbano y rural) y de la titularidad del centro (público o concertado/ privado). En la segunda fase, se reparó en la variable 'sexo' como aspecto reseñable desde el punto de vista de la selección muestral.

Según datos del Instituto Nacional de Estadística (INE, 2008), la población gallega entre los 11 y los 12 años ascendía a 41.889 personas (21.513 hombres, 20.376 mujeres) repartidos en 898 centros públicos y privados que imparten enseñanza primaria. Teniendo en cuenta el universo definido, para un índice de confianza del $95,5 \%$ con un margen de error de $+/-2,5 \%$, la muestra para ser representativa, debía estar formada por 1.542 individuos.

DOI: ri14.v13i1.739 | ISSN: 1697-8293 | Año 2015 Volumen 13 Nº 1 | ICONO14 


\begin{tabular}{|c|c|c|c|}
\hline 10 a 12 años & TOTAL & HOMBRES & MUJERES \\
\hline A CORUÑA & 16.635 & 8.467 & 8.168 \\
\hline LUG0 & 4.670 & 2.355 & 2.315 \\
\hline OURENSE & 4.601 & 2.405 & 2.196 \\
\hline PONTEVEDRA & 15.983 & 8.286 & 7.697 \\
\hline TOTAL GALICIA & 41.889 & 21.513 & 20.376 \\
\hline
\end{tabular}

Tabla 1: Población de 11 y 12 años en Galicia. Fuente: INE, 2008

\begin{tabular}{|c|c|c|c|c|}
\hline Centros educativos & TOTAL & CEIP & CEP $^{2}$ & PRIVAD0 \\
\hline A CORUÑA & 330 & 221 & 6 & 103 \\
\hline LUG0 & 124 & 93 & 1 & 30 \\
\hline OURENSE & 120 & 84 & 0 & 36 \\
\hline PONTEVEDRA & 324 & 197 & 16 & 111 \\
\hline TOTAL GALICIA & 898 & 595 & 23 & 280 \\
\hline
\end{tabular}

Tabla 2: Centros educativos de Galicia que imparten enseñanza primaria.

Fuente: Consellería de Educación, A Xunta de Galicia, 2010

Una vez conseguido el consentimiento de los colegios y tras realizar el trabajo de campo, se contabilizó en la base de datos -se empleó SPSS para el tratamiento estadístico- una muestra final de 2.200 individuos pertenecientes a 77 centros públicos y privados de enseñanza primaria de las cuatro provincias de la comunidad gallega (31 ubicados en A Coruña, 29 en Pontevedra, 9 en Ourense y 8 en Lugo).

Siguiendo a Sierra Bravo (2001:305) el instrumento básico de la observación por encuesta es el cuestionario que puede ser de tres clases: cuestionario sim- 
ple, entrevista o escalas sociométricas. En este estudio se recurrió al cuestionario simple con el que los encuestados, previa su lectura, contestaron por escrito sin intervención directa de las personas que colaboran en la investigación. De forma anónima, los estudiantes rellenaron una encuesta de 25 preguntas, la mayoría de respuesta cerrada a excepción de tres en las que se buscaba que los niños y niñas escribiesen cuáles eran sus cadenas de televisión, videojuegos y sitios web favoritos. Dicho cuestionario buscaba conocer el grado de equipamiento, las horas de conexión y los contenidos a los que accede la muestra seleccionada en relación a las tres pantallas de estudio, televisión, internet y videojuegos.

\section{Resultados}

En este apartado se presenta una descripción general de las rutinas de los más pequeños por internet al analizar las horas de conexión tanto durante los días de colegio como en fin de semana y sus sitios web favoritos, es decir, qué consumen cuándo están conectados, con una especial atención al nivel de preferencia por las redes sociales y la prensa digital.

\subsection{Horas de conexión: días lectivos y fines de semana}

La mayoría de la muestra, un $67,6 \%$ reconoce conectarse a internet menos de una hora durante los días de colegio; un 17,9\% lo hace de una a dos horas y menos de un 7\% admite estar más de dos horas. El fin de semana la tendencia cambia y los resultados muestran cómo los periodos de más tiempo de conexión ganan presencia a cuesta de los de menor tiempo. Los niños que se conectan menos de una hora se reducen al 37,5\% (a menos de la mitad con respecto a la semana) y se incrementan en más del doble el resto de opciones. De este modo, un $43,3 \%$ de la muestra navega por la red de una a tres horas sábados y domingos y aproximadamente el 55\% reconoce hacerlo al menos por una hora. En esencia, mientras que el consumo de internet se concentra en menos de una hora durante los días de colegio, los niños aprovechan el fin de semana para pasar más tiempo conectados.

DOI: ri14.v13i1.739 | ISSN: 1697-8293 | Año 2015 Volumen 13 Nº 1 | ICONO14 

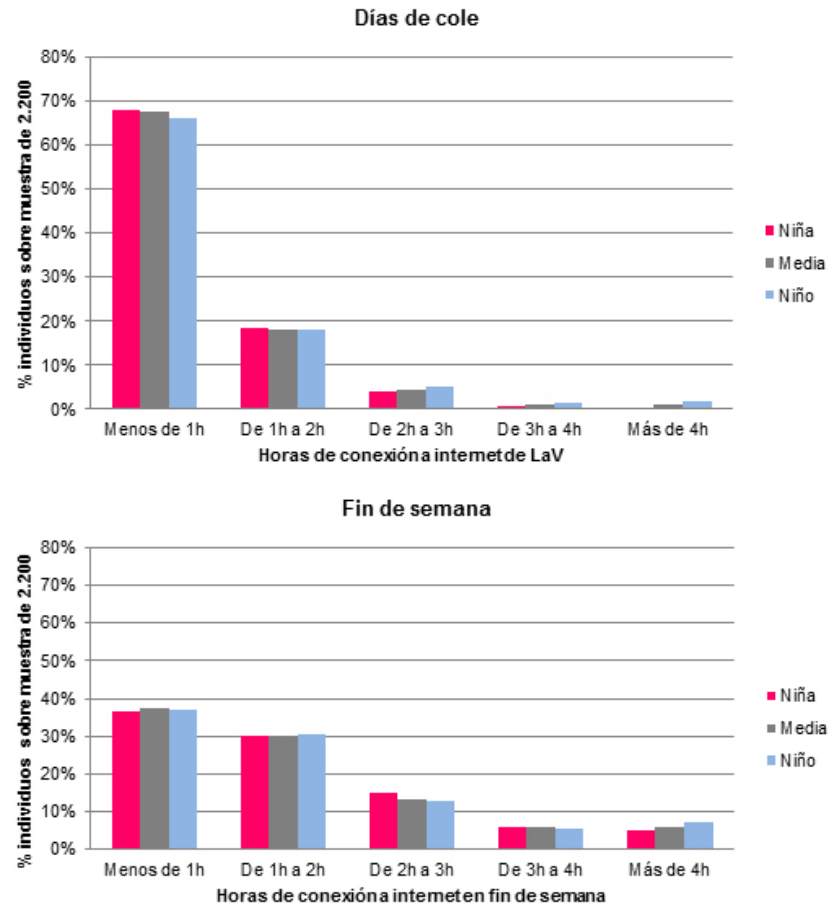

Figura 6: Horas de conexión a internet diferenciadas por días de la semana y sexo. Fuente: Elaboración propia, 2014

Si se atiende al sexo no se aprecian diferencias significativas, aún así los niños tienden a pasar más número de horas conectados, sobre todo durante la semana.

\subsection{Espacios web favoritos}

Las acciones que niños y niñas suelen desempeñar por la red se pueden resumir en tres: buscan entretenerse, compartir y comunicarse con sus iguales $\mathrm{y}$, en tercer lugar, también lo usan para aprender y conocer. Los espacios web favoritos de los encuestados son los de juegos online, seleccionados por casi siete de cada 10 niños. Las redes sociales $(52,8 \%)$ y las páginas contenedoras de vídeos y música (39\%) también ocupan una posición preferente en los gustos infantiles. Las visitas a espacios más relacionados con el aprendizaje o el conocimiento, aunque son incluidos en sus elecciones, cuentan con porcentajes inferiores. Las webs corporativas - sobre todo las oficiales de personajes de 
series y dibujos animados-, los buscadores (Google principalmente) y la prensa digital son algunos de los espacios más nombrados dentro de esta categoría.

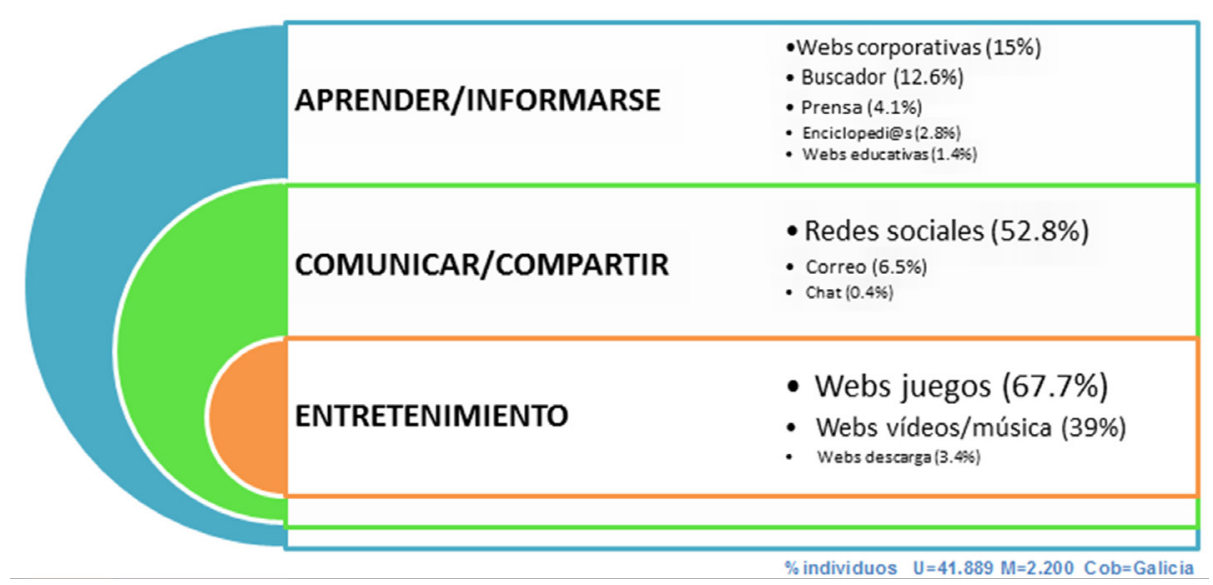

Figura 7: Espacios web más visitados. Fuente: Elaboración propia, 2014

Los niños manifiestan una mayor predilección por las webs de juegos, las niñas, por su parte, optan más por emplear las redes sociales. Como dato curioso se presenta la afinidad detectada entre los niños y la prensa online (sobre todo la deportiva) y entre las niñas y las web corporativas (de personajes de series y dibujos infantiles).

\subsection{Redes sociales Vs. prensa digital}

Si se compara en nivel de penetración de las redes sociales y prensa digital en las rutinas digitales de los encuestados, por cada niño que visita prensa digital, casi 13 están en alguna red social.

Aunque pueda parecer un resultado desigual, hay que tener en cuenta que la muestra escogió sus espacios web favoritos a partir de una pregunta abierta. Las opciones citadas fueron mencionadas libremente por los encuestados, recordadas sin ningún tipo de guía previa, fruto de su memoria y de su short list virtual y la prensa digital sí se encuentra entre los diez espacios web más nombrados por la muestra.

DOI: ri14.v13i1.739 | ISSN: 1697-8293 | Año 2015 Volumen 13 N 1 | ICONO14 


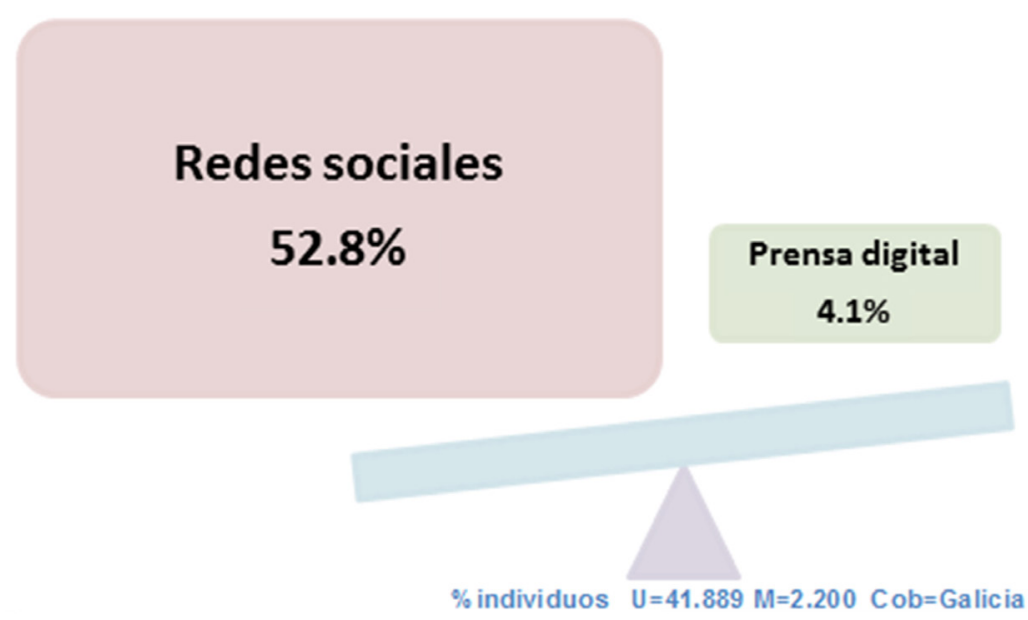

Figura 8: Comparativa penetración redes sociales y prensa digital.

Fuente: Elaboración propia, 2014

Si se diferencia por sexo aparecen algunas diferencias. Por su parte, las redes sociales cuentan con una mayor penetración entre las niñas, cuyo porcentaje roza el $62 \%$ frente al $44,5 \%$ conseguido entre los niños. En cambio, a la prensa digital le ocurre totalmente lo contrario, los lectores masculinos son sus más fieles seguidores: en este perfil, el diario digital logra una penetración del 7\% (casi el doble de la media), mientras que entre las niñas no llega al 1\%. El motivo, la prensa deportiva: en esta categoría, los principales espacios web recordados fueron las versiones digitales de los diarios Marca, As y Sport.

Si se analiza un poco más en profundidad el nivel de penetración de las redes sociales, los encuestados también tienen sus favoritas. Windows con sus servicios Messenger son los que consiguieron una mayor presencia al ser recordados por un 35,8\% de la muestra. Tuenti con un 24,4\% y Facebook con un $13,3 \%$ ocupan la segunda y tercera posición aunque no permitan en sus condiciones legales el acceso a menores de estas edades objeto de estudio (11-12 años). Las redes sociales fotográficas como Metroflog y Fotolog que últimamente han perdido seguimiento entre los usuarios más jóvenes, completan el Top 5. Finalmente Habbo, pensada para adolescentes, cierra la lista con un 4,1\% de penetración. 


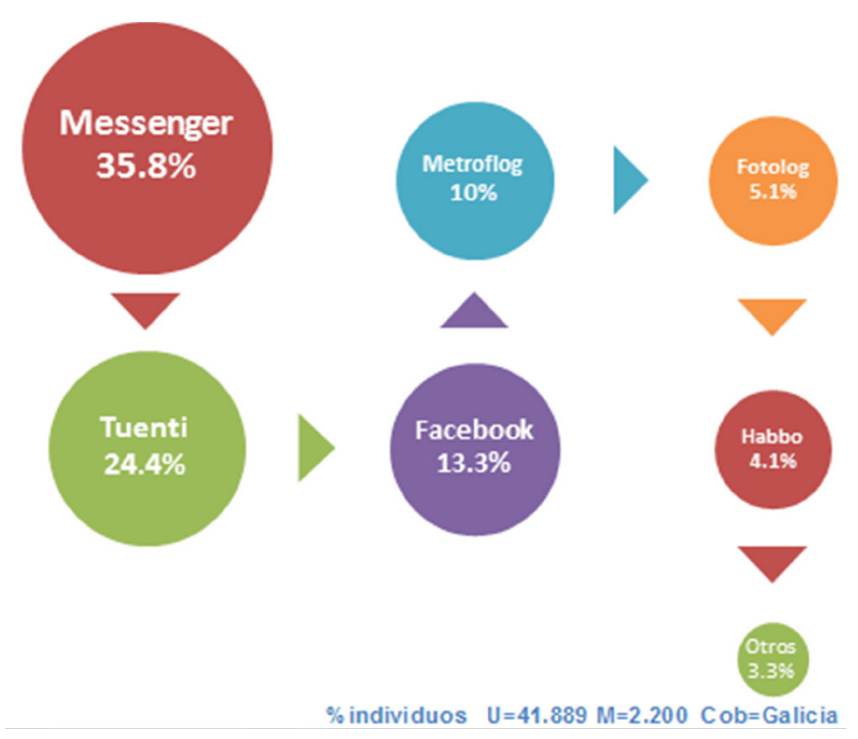

Figura 9: Redes sociales más citadas. Fuente: Elaboración propia, 2014

\section{Discusión}

Las redes sociales cuentan con un mayor seguimiento y fidelidad por parte de la muestra que la prensa digital: un $52,8 \%$ de uso frente al $4,1 \%$ de los diarios. Puede que sea un resultado esperable porque las redes sociales se basan en explotar una actividad que los más pequeños buscan y desarrollan en esas edades, la de socializarse y establecer contacto con iguales. Tras los videojuegos online, la red social es el recurso web más visitado, aunque legalmente este público tenga el acceso prohibido por no alcanzar la edad mínima para abrir un perfil, 14 años.

Aunque ninguno de los dos espacios se concibió inicialmente para un usuario infantil, la esencia de las redes sociales hace que el diario digital no pueda competir al mismo nivel. No obstante, este estudio pone de manifiesto que, al margen de comparaciones, la prensa online no es ajena completamente a los niños y niñas.

A lo largo de este artículo se mencionó como la prensa en general y la digital en particular han puesto en marcha proyectos con una clara vocación didáctica con 
el objetivo de atraer la atención de los más pequeños hacia el medio. No obstante, los resultados de esta investigación muestran que, aunque el diario online se encuentra entre los 10 sitios web citados por la muestra, no se puede considerar una actividad preferente en las rutinas digitales de estos niños y niñas. De hecho, más individuos optan por acceder a información a través del buscador (principalmente Google) o de páginas corporativas que a través de la prensa. Como fuente de consulta no es prioritaria y ocupa un posicionamiento próximo a las enciclopedias virtuales, cada vez más en desuso.

Por tanto, parece evidenciarse que las acciones diseñadas por el medio prensa para acercarse al estudiantado infantil no aparecen reflejadas en las rutinas digitales de muestra referente para esta investigación. Es más, a pesar de la perspectiva pedagógica de estos proyectos de prensa-escuela, es más bien escaso el compromiso de los centros docentes con este tipo de iniciativas posiblemente motivado por el estancamiento de la enseñanza en medios de comunicación (Ricoy, 2009).

A modo de discusión, puede que la falta de conexión entre el público infantil y la prensa sea consecuencia de que los propios diarios conciban estas iniciativas dirigidas a niños más como una estrategia de responsabilidad corporativa que como una acción para captar nuevos perfiles de lectores. Esto unido a una falta de colaboración de las escuelas en integrar la prensa como un recurso habitual en las aulas puede que sean los motivos de su baja presencia en las rutinas de los más pequeños, una cuestión que se propone, pero que requeriría de una reflexión mucho más profunda.

\section{Notas}

[1] CEIP, acrónimo de Centro de Enseñanza Infantil y Primaria

[2] CEP, acrónimo de Centro de Enseñanza Primaria

ICONO14 | Año 2015 Volumen $13 N^{\circ} 1$ | ISSN: 1697-8293 | DOI: ri14.v13i1.739 


\section{References}

Bringué, X. \& Sádaba, C. (2009). La generación interactiva en España. Niños y adolescentes ante las pantallas. Fundación Telefónica y Editorial Ariel, Madrid.

Bringué, X. \& Sádaba, C. (2011). Menores y redes sociales, Foro Generaciones Interactivas, Universidad de Navarra.

Bringué, X., Del Río, J. \& Sádaba, C. (2008). Menores y redes ¿sociales?: de la amistad al cyberbulling. Departamento de Comunicación Audiovisual, Universidad de Navarra.

Buil, I., Hernández, B. \& Sesé, F.J. (2013). “El papel de la prensa en la mejora del proceso de aprendizaje. Una aplicación en el EEES". En Revista de Docencia Universitaria, vol.11, n³, pp.445-459.

Cea D’Ancona, M.A. (1996). Metodología cuantitativa: estrategias y técnicas de investigación social. Madrid: Síntesis, D.L.

García Fernández, F. (2010). Las redes sociales en la vida de tus hij@s. Cómo conseguir que se relacionen on-line de forma segura y responsable. Abertis. INTECO (2010). Redes sociales, menores de edad y privacidad en la red. Artículo realizado por el Instituto Nacional de Tecnologías de la Comunicación. Observatorio de la seguridad de la información. Área jurídica de la Seguridad y las TIC.

Libro Blanco de la Prensa Diaria 2013. Madrid: Asociación de Editores de Diarios Españoles.

Monsoriu Flor, M. (2009). Manual de redes sociales en Internet: aprende a usar Tuenti, Facebook, Fotolog, Myspace, etc., ¡Mejor que tus hijos! Creaciones Copyright.

Prensky, M. (2001). Digital Natives, Digital Immigrants. On the Horizon (MCB University Press), Vol. 9, 6. Recuperado de http://goo.gl/zYLOy1

Ricoy, M.C. (2006). “La prensa como recurso en el curriculum de adultos”. En Comunicar, vol.14, n²6, pp. 199-206.

Ricoy, M. C. (2009). “Utilización de los recursos y factores que rodean el empleo de la prensa en la educación de jóvenes y adultos". En Zer, vol.14, n²6, pp.145-166.

Romano, V. (2003). “Educación ciudadana y medios de comunicación". En Revista 
de Educación, ${ }^{0}$ extra, pp. 391-401.

Sierra Bravo, R. (2001). Técnicas de Investigación Social, Teorías y ejercicios. Paraninfo.

Recursos electrónicos

V Estudio anual sobre Redes Sociales: http://www.iabspain.net/redes-sociales/ (Último acceso: 20 septiembre de2014).

Asociación para la investigación de medios de comunicación (AIMC): http://www. aimc.es (Último acceso: 5 de septiembre de 2014).

Comunicación y Medios: http://www.comunicacionymedios.com/comunicacion/ teorias/textos/internet.htm (Último acceso: 7 de septiembre de 2014).

Foro Generaciones Interactivas: http://www.generacionesinteractivas.org (Último acceso: 30 de agosto de 2014)

Media-tics. Información y comunicación en la era digital: http://www.media-tics. com/noticia/2504/Medios-de Comunicacion/Periodicos-infantiles:-inversionen-futuro.html (Último acceso: 27 de septiembre de 2014)

0JD Interactiva: http://www.ojdinteractiva.es/ (Último acceso: 5 de septiembre de 2014).

Resumen informe Spain digital future in focus 2013: http://es.slideshare.net/ XoseM/spain-digital-future-in-focus-2013 (Último acceso: 30 de septiembre de 2014).

Uso de las redes sociales en España y resto del mundo. http://www.youtube.com/ watch?v=uitAUu7cVSw (Último acceso: 28 de agosto de 2014).

Xatakaciencia: http://www.xatakaciencia.com/sabias-que/la-teoria-de-los-seisgrados-de-separacion (Último acceso: 2 de septiembre de 2014).

ICONO14 | Año 2015 Volumen 13 Nº 1 | ISSN: 1697-8293 | DOI: ri14.v13i1.739 